\title{
Exhausting, pneumatic transport
}

\section{Karel Adamek ${ }^{1}$, Jan Kolar ${ }^{1}$, Pavel Peukert ${ }^{1}$, Martin Pustka $^{1}$, Petr Pulpan $^{1}$, Radim Foldyna ${ }^{2}$, Petr Bilek $^{3}$, Ales Prusek ${ }^{4}$}

${ }^{1}$ VUTS - Centre of Machinery Research, Liberec, Czech Rep

${ }^{2} \mathrm{HP}$ - Pelzer, Zatec, Czech Rep

${ }^{3}$ Preciosa, Jablonec nad Nisou, Czech Rep

${ }^{4}$ Magna- Exteriors and Interiors Bohemia, Liberec, Czech Rep

\section{Email address:}

karel.adamek@vuts.cz (K. Adamek), jan.kolar@vuts.cz (J. Kolar), pavel.peukert@vuts.cz (P. Peukert), martin.pustka@vuts.cz (M. Pustka), petr.pulpan@vuts.cz (P. Pulpan), r.foldyna@ pelzer-mb2.cz (R. Foldyna), petr.bilek@preciosa.com (P. Bilek), a.prusek@eu.magna.com (A. Prusek)

\section{To cite this article:}

Karel Adamek, Jan Kolar, Pavel Peukert, Martin Pustka, Petr Pulpan, Radim Foldyna, Petr Bilek, Ales Prusek. Exhausting, Pneumatic Transport. International Journal of Mechanical Engineering and Applications. Special Issue: Moving Forward to Monitory Democracy: Citizens Engagement in Scrutinizing Election Process in Indonesian 2014 General Election. Vol. 3, No. 1-1, 2015, pp. 33-39. doi: 10.11648/j.ijmea.s.2015030101.16

\begin{abstract}
The contribution describes frequent functional problems of exhausting devices, caused by no observing of essential rules of fluid mechanics. For instance, due to the unbalanced tubing system of several branches the medium flows in individual connections and their relevant energy transports are not corresponding to the designed one, or the diffuser of very large enlargement leads to the flow separation, increased noise level etc.
\end{abstract}

Keywords: Numerical Flow Simulation, Exhausting, Pneumatic Transport, Aerodynamic Noise

\section{Introduction}

The operational problems of many technical devices and equipment consist in a bad design from the point of view of fluid mechanics. This text presents some examples.

\section{Exhausting Systems}

\subsection{Common Exhausting Channel}

The frequent operational problem is the equalizing of the suction or of an inlet system of many working units, connected with one common suction or inlet conduit. One typical problem is the flow in the collecting channel for exhaust of impurities on spinning machine, equipped by many units. Fig. 2.1-1 shows the flow field at the beginning of such a channel with common exhaust situated on the right side. To equalize the flow field in the inlet cross section, it should be enlarged the right side of the common channel (not presented here). If all connections have the same cross sections, it is clear that in the furthermost one the flow will be the smallest and it is possible that in this area, some sedimentation of transported impurities arises if the velocity value is under the so-called buoyancy velocity.

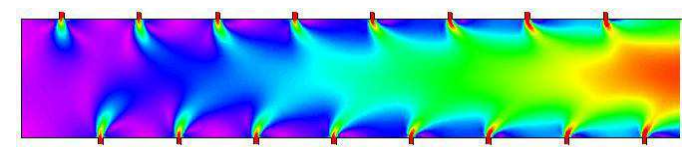

Figure 2.1-1. Velocity isolines in common exhaust channel of many individual connections

To equalize the volumes exhausted from individual connections it should be either to use graded cross sections of individual connections, i.e. the furthermost with the largest cross section, or to increase the pressure loss in the nearest connections. This idea can be realized by iterating method in several steps - the solved numerical model of given geometry is evaluated, after the evaluation, some cross sections (their pressure resistances) are changed and the changed model is simulated again. The procedure finishes when difference of the results in the last two steps is less than the acceptable value.

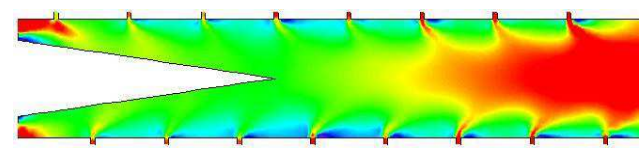

Figure 2.1-2. Increased velocity in common exhaust channel after cross section decreasing 
On the detailed view in Fig. 2.1-3, it is visible that inlet flows from individual connections are acting as rigid obstructions in the main axial flow.

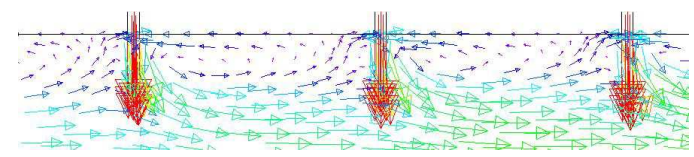

Figure 2.1-3. Detail of streamlines from individual connections

\subsection{Unbalanced Exhaust System}

In Fig. 2.2-1, there is presented the geometry and pressure field of a complicated suction system of 8 connections into one common exhaust. But the system operation is unsatisfactory, the aim of the solution is to find the reason and to define the remedy [1].

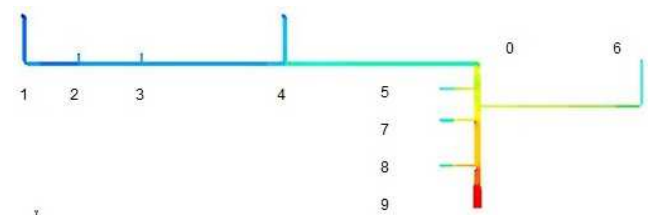

Figure 2.2-1. Scheme of solved exhaust system with pressure distribution

The solution of the numerical model with designed values (lengths, diameters, volume flows) has very uneven flows distribution, see the profile „1“ in Fig. 2.2-2. Because the flow volumes in individual connections are very different, the graph presents the relative flow of each connection as ratios of the real (simulated) flow to the designed one ( $\mathrm{ms} / \mathrm{mp}$ ).

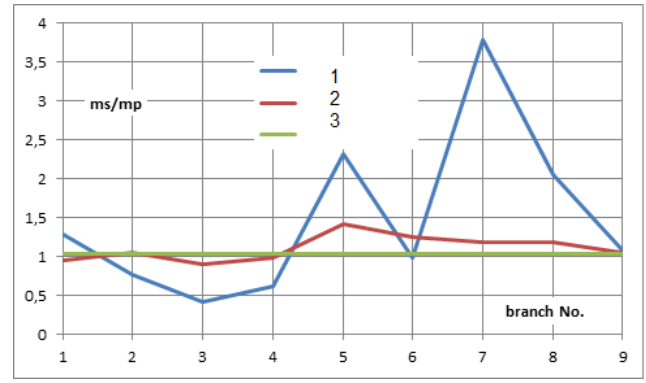

Figure 2.2-2. Flow profiles after step-by-step improving

The profile No. 1 represents the initial design after the project. It is clear that flows are tuned not at all.

Decreasing the cross sections in the connections of high flow and on the contrary, increasing the cross sections in the connections of insufficient flow, the common profile is better balanced, see the profile No. 2.

In next iterations, the individual pressure resistances at inlets into individual connections were exactly balanced to get all flows after the project, see the profile No. 3. During such tuning, it is necessary to take into account that some flow change in one connection influences flows in the neighboring connections, too.

By next simulations of flow in flaps of appropriate diameters at various flap positions $\left(^{\circ}\right)$, the appropriate pressure resistances $(\mathrm{Pa})$ were assigned. As an information,
Fig. 2.2-3 and Fig. 2.2-4 show a pressure and a velocity field for one flap position, only.

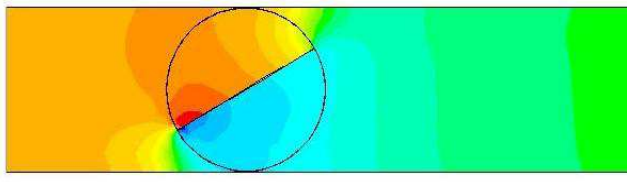

Figure 2.2-3. Pressure field at one flap position.

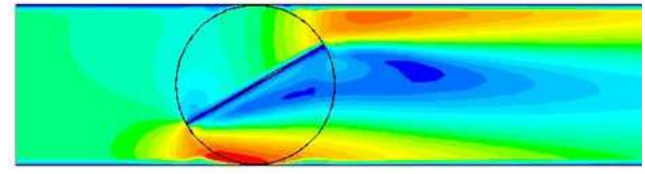

Figure 2.2-4. Velocity field at one flap position.

For the sets of flap position, there were evaluated flap flow characteristics, as an example see Fig. 2.2-5.

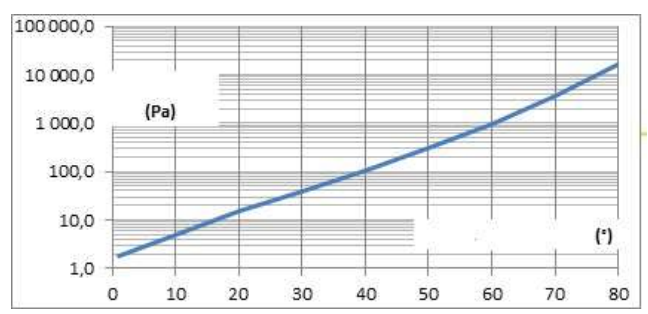

Figure 2.2-5. Flap characteristics - resistance $(\mathrm{Pa})$ as function of position $\left(^{\circ}\right)$

The use of the method of numerical flow simulation in such a complicated tube system is very profitable. Even it is possible to state that the classical method of calculation of pressure losses as the sum of resistances in straight parts with inserted so-called local losses is not suitable.

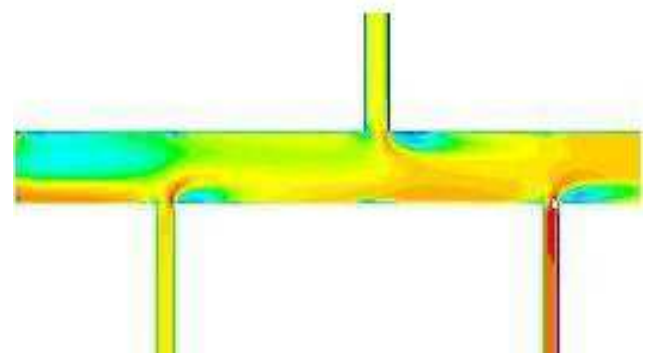

Figure 2.2-6. Mutual affecting of velocity fields in connections close together

In Fig. 2.2-6, there is visible that the inflow effect in one connection of the flow takes effect in the adjoining one, too. The same image is possible to use for determination of the right positioning of measuring devices - measuring should be realized in the area of undisturbed flow.

\subsection{Noise and Vibrations}

The velocity field of the actual design is presented in Fig. 2.3-1. In the complicated cornered shape, there are visible large areas of the flow separation with backflow, the consequence of it is the oscillating pressure field. 


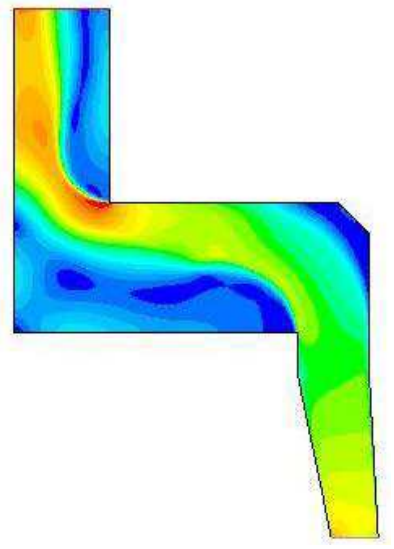

Figure 2.3-1. Velocity field - actual shape

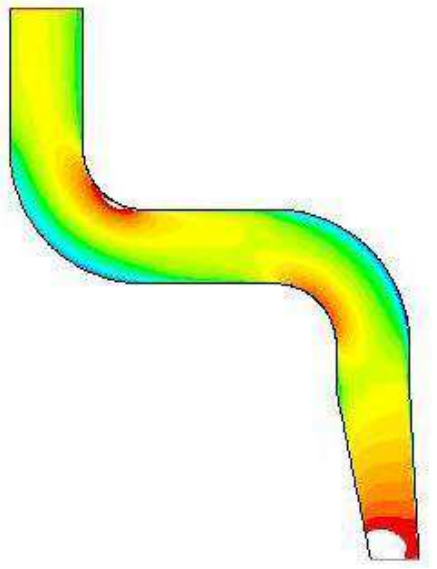

Figure 2.3-2. Velocity field - new design

After this image of the main flow, the new design after Fig. 2.3-2 was realized, where the areas of flow separation are minimal, only and could be suppressed yet by next small modifications [2], [3].

For verification of the hypothesis on the vertical wall of the horizontal part of the system, the oscillations of the simulated flow field were recorded. The record is displayed in Fig. 2.3-3.

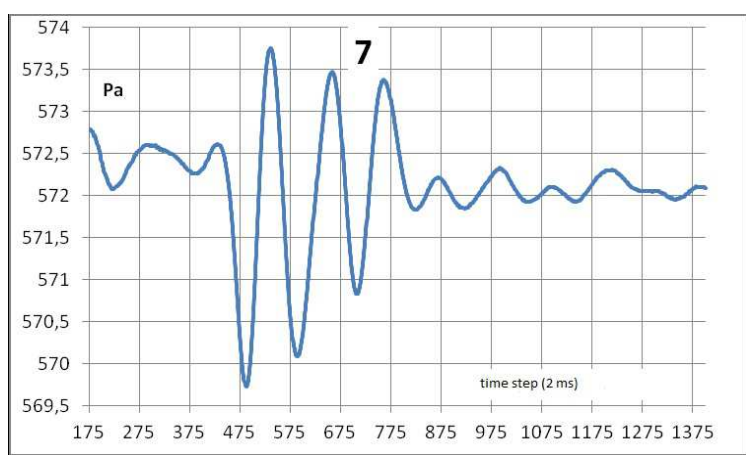

Figure 2.3-3. Pressure oscillations on original shape

The frequency analysis of this signal is presented in Fig. 2.3-4, the maximum value of acoustic pressure is $0.5 \mathrm{~Pa}$ at a frequency of $4.5 \mathrm{~Hz}$. This is in exact correspondence with the really measured values. The force effect of it could be theoretically presented as the effect of the mass of $0.6 \mathrm{~kg}$, falling 5 times per second onto the thin metallic area of $12 \mathrm{~m}^{2}$ - really ramble!

Remark: Another pressure oscillations were detected from the blade wheel rotation, here are presented the aerodynamic ones, only.

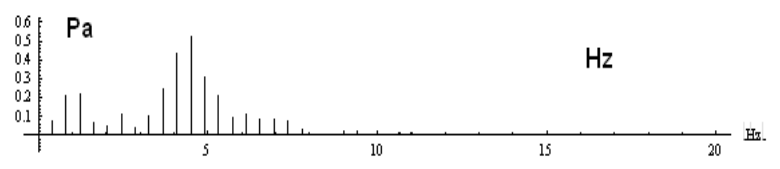

Figure 2.3-4. Frequency analysis of the signal from the Fig. 2.3-2

In several points of the new design from Fig. 2.3-2, the oscillations of the simulated flow field are recorded in Fig. 2.3-5. After a short starting period of the numerical simulation, the flow field does not contain any pressure oscillations.

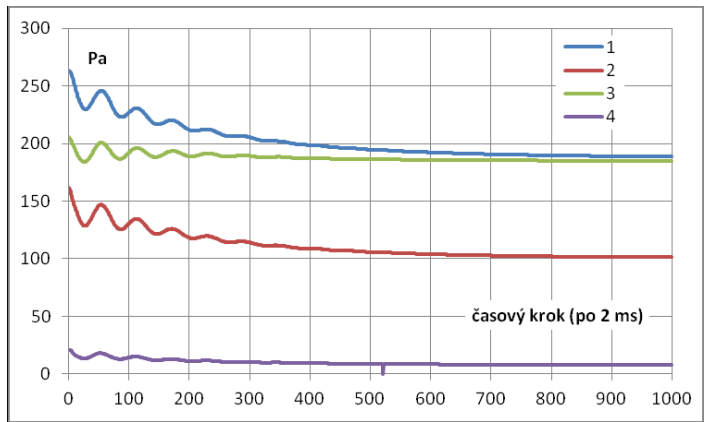

Figure 2.3-5. Pressure oscillations on modified shape

The mentioned pressure oscillations of the flow field in the given geometry call out secondary oscillations of the large and thin metallic walls of the system, generating further the noise into the surroundings.

The simplest solution there is the straight outlet up, finished by a suitable damper, made from rigid material, for instance after Fig. 2.3-6 [4].

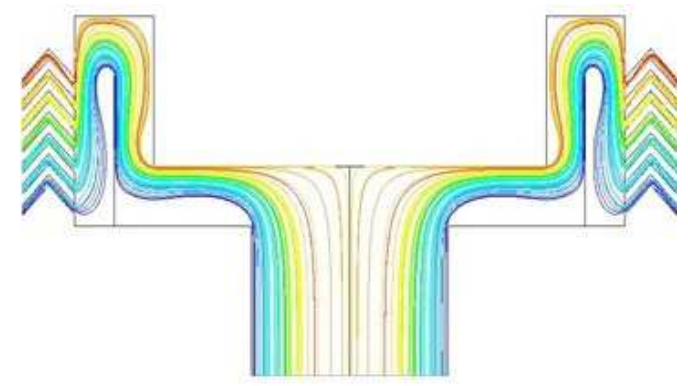

Figure 2.3-6. Streamlines in simple and cleanable labyrinth noise damper

\section{Pneumatic Transport}

\subsection{Chips Transport}

The rotating knife head after Fig. 3.1-1 is shaping the face of the pressed part and the arising chips are exhausted by air flow.

The pressure field around the rotating head, as found by numerical flow simulation, has not any important influence on 
total air flow.

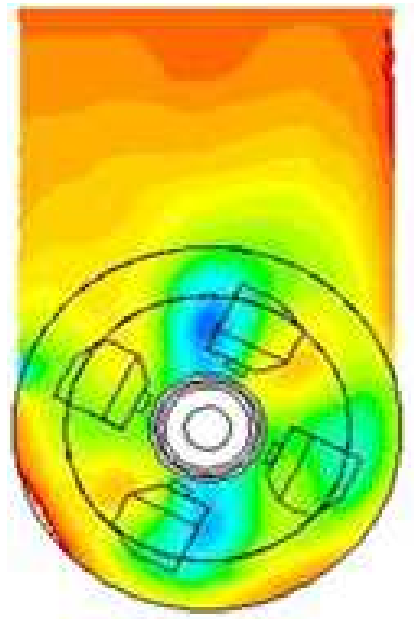

Figure 3.1-1. Pressure field in the vicinity of rotating head (axial view)
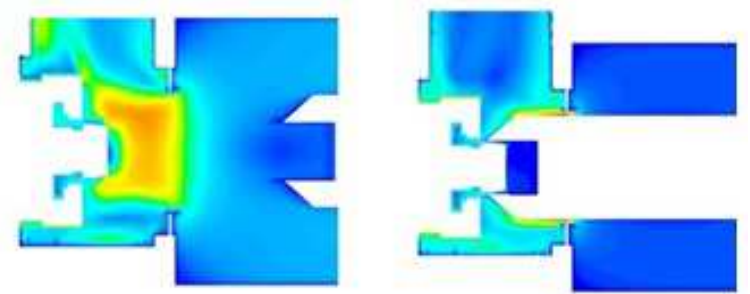

Figure 3.1-2. Pressure field in the vicinity of rotating head-left: part is out, right: part is in

The next Fig. 3.1-2 presents the velocity field in the lengthwise cross section, on the left the shaped part is out and on the right is in and just shaped.

On the first view it is visible that by inserting the part into the opening (here from right) the air inlet is clogged so that the medium air velocity in the exhaust pipe is decreasing under the so-called buoyancy velocity, which should have $15-20 \mathrm{~m} / \mathrm{s}$ as the recommended minimum for transport of metallic chips.

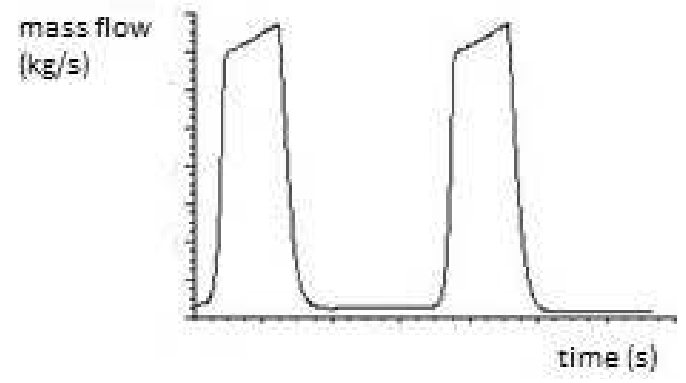

Figure 3.1-3. Mass flow during working cycles

The corresponding air flow is presented in Fig. 3.1-3. It is clear that by the clogging of the inlet opening by the shaped part, the velocity is decreasing significantly.

The next graph in Fig. 3.1-4 shows in relative values the under pressure at the inlet and its delayed values at the outlet, too - for instance the full flow at the full opening is reached with some delay after the inlet opening. Except the pressure loss, here it is expressed the transporting delay, given by both distance and volume between inlet and outlet.

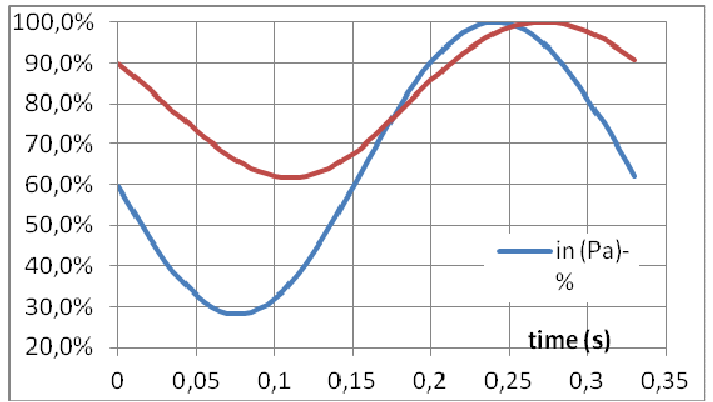

Figure 3.1-4. Pressure course at inlet (blue) and outlet (red)-relative values

\subsection{Production of Insulating Panels}

The principle of the insulating panel production for the car industry consists from the insulating material transport (yarns, chips) by air flow. Finally the material is settling on the perforated surface of the die, corresponding to the designed shape of product. After sedimentation of sufficient layer, the material is pressed and heated. The manufactured insulating panel has the sufficient tenacity for next treatment, transport and assembling.

In Fig. 3.2-1, there is the velocity and directional fields in such a forming chamber - the air inlet together with material is situated from the left side, in the chamber, the material is dispersed and settled on the perforated form on the chamber ceiling.

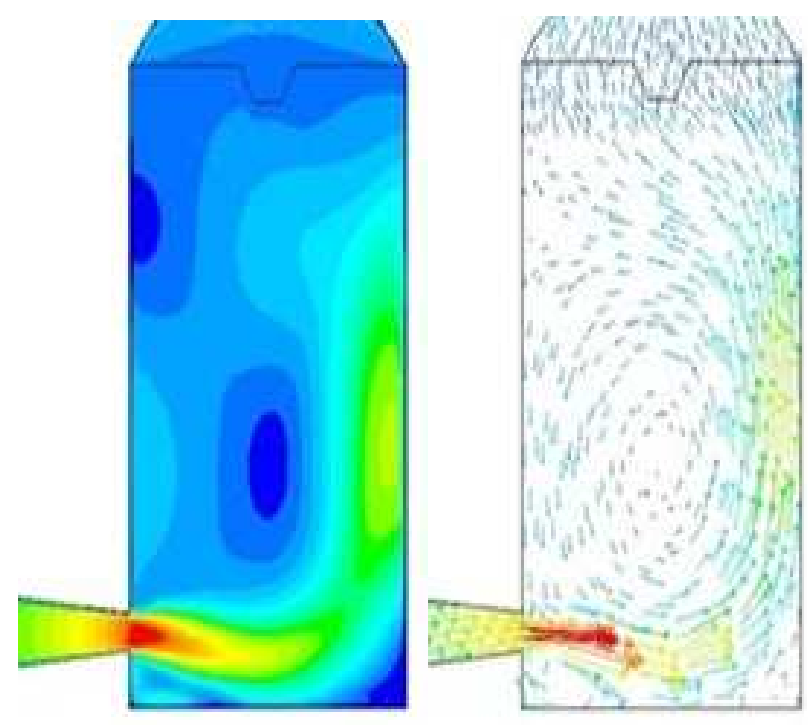

Figure 3.2-1. Velocity and directional field in chamber

Generally, it is visible that the material sedimentation on the form surface is not fully uniform and that it will be necessary to adjust the flow image by any inserted partitions to get the required material dispersion on the form surface - either fully uniform or with thinner periphery, after the demands of the design.

\subsection{Suction Head}

The suction head is a frequent device of mechanization or 
automation in many applications - suction, exhaust, sucker, etc. The necessary under pressure is reached for instance by the suction effect of an ejector, exhauster, etc.
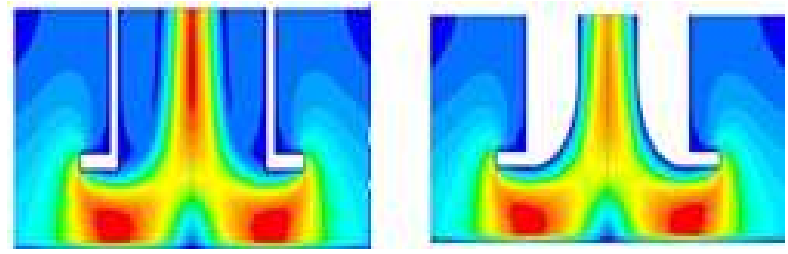

Figure 3.3-1. Velocity field in suction head

On the left side of Fig. 3.3-1, there is the velocity field in a possible design of suction head. It is well-known that the suction effect is increasing when the suction outlet is provided by a flange - thereby the suction is realized from one half-space, only, and the suction effect is increasing (here is the max. value of $38 \mathrm{~m} / \mathrm{s}$ ). On the right side of Fig. 3.3-1, there is an adjustment of the inner shape of the suction head - by suitable rounding the inner area of separation is removed and by a generally better image of the flow field, the suction effect will be better, too (max. value of $45 \mathrm{~m} / \mathrm{s}$ ).

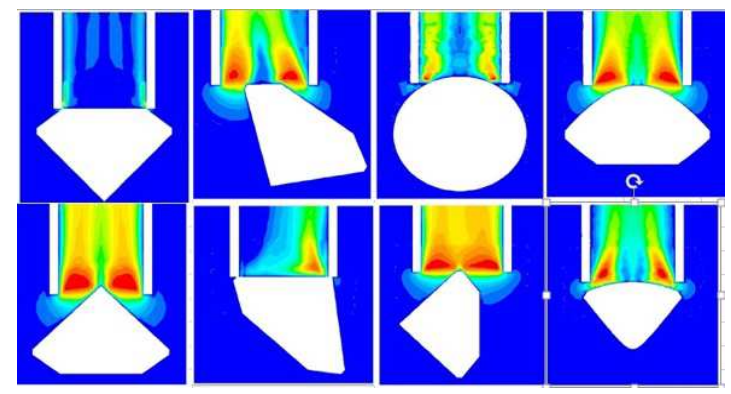

Figure 3.3-2. Velocity field for various part positions toward the suction head inlet

The next serial in Fig. 3.3-2 presents the velocity fields for a possible application of the suction head: to catch and transport a part of complicated shape. At first, for the catching of the part, it is necessary to create a sufficient suction effect, which is fading relative expressively with the distance between the part and suction head opening. Once the part is caught up, for its holding it is enough to use a smaller exhaust flow. After the actual situation, it is possible to operate such a suction head in two modes of different flows and thereby to decrease the total consumption of air and energy. Evaluating such numerically simulated situations, it is possible to determine the necessary suction force for the actual part position, depending on the under pressure or on the air flow and to determine the air consumption in advance.

\subsection{Transport and Drying of Parts}

The following two applications are from the food industry. The first presents the velocity field from two parallel orifices around the cylindrical body. The device is used for a common drying and transport of such a cylinder, using hot flows from two long and narrow gaps.

Fig. 3.4-1 shows the ideal coaxial position, the body position is determined as the result of cylinder weight and of the aerodynamic force of air flows on the cylinder surface which are fading with the increasing cylinder distance from the nozzles. At the given air pressure in the inlet, some stable distance in height is created.

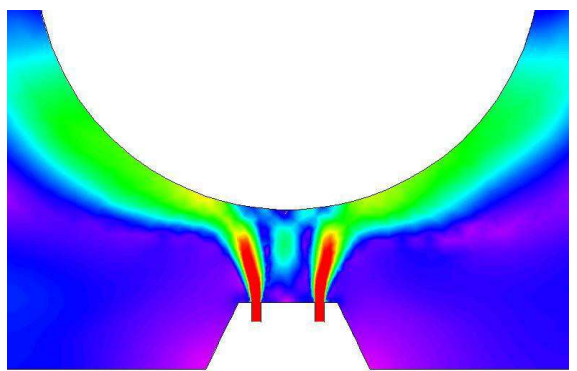

Figure 3.4-1. Velocity field - coaxial position of cylinder

In Fig. 3.4-2, there is presented some offset position, given for instance by any effect of external forces. The vector of aerodynamic force (both direction and size), forcing onto the cylinder, it is possible to evaluate from the pressure distribution on the cylindrical surface. From the balance of force moments (both aerodynamic and gravitational),it is possible to determine, if such position is stable, it means if it returns back into the coaxial position or if it is unstable, it means that the cylinder is falling out from the forcing area of air flows from the nozzles. In such a case, it is necessary to change the nozzle arrangement and air pressure.

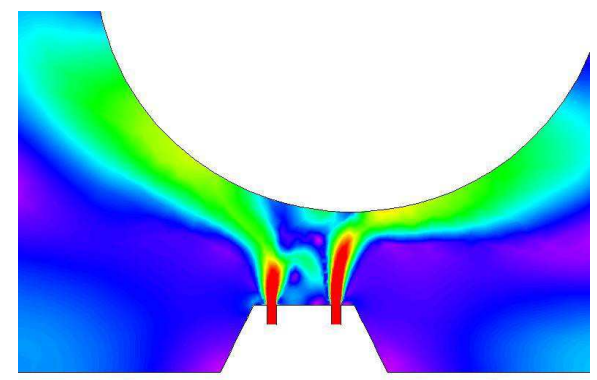

Figure 3.4-2. Velocity field - cylinder offset

The next case shows similar situation, but the dried and transported part has the form of toroid [5]. The body is carried and dried by several small flows, acting on the inner surface of the toroid; their effect is expressed by the dynamic pressure field, which must be balanced with the toroid weight.

The relevant fields of dynamic pressure (left) and of temperature (right) in Fig. 3.4-3 have the main influence on the drying intensity, except of the surface velocity, too. Here it is visible that the lower outer surface of the part is not well heated.

Adding the next air flow from the outer lower side, the temperature and the drying intensity is increasing, see Fig. $3.4-4$, but in the same time the buoyant force is increasing, which has a destabilizing effect. In any case, the stable balance of all actuating forces - the aerodynamic and gravitational must be kept. 


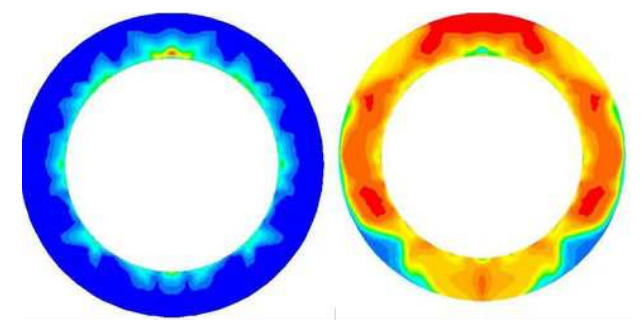

Figure 3.4-3. Dynamic pressure (left) and temperature (right) on toroid surface-former design
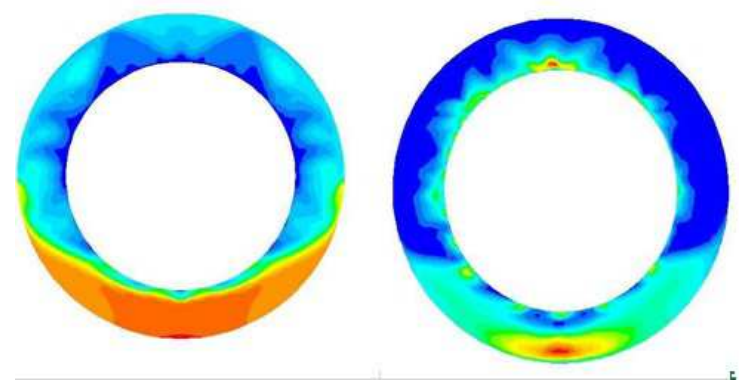

Figure 3.4-4. Dynamic pressure (left) and temperature (right) on toroid surface - adapted design

\section{Burners}

Problems of uniform flows were detected in the solved cases of burners as follows.

\subsection{Diffuser}

Gas burners used in glass industry have the shape of diffuser after Fig. 4.1-1. Of course, in such rush enlargement of the cross section, the flow is separating from the walls and the backflow in the shape of long vortex brings some impurities inwards (here not displayed), which are sediment on the inner surface and make some operational troubles.

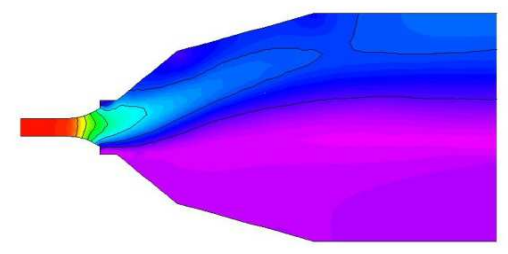

Figure 4.1-1. Diffuser of rush enlargement.

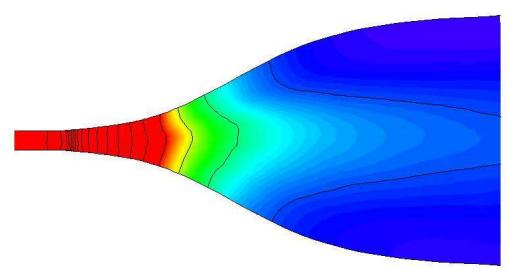

Figure 4.1-2. Diffuser of fluent enlargement

It is well-known that a suitable value of diffuser enlargement should not exceed $10-12^{\circ}$. Even if it is not possible, at the given main dimensions of the diffuser (inlet and outlet diameters, length), to comply with this condition, it is possible to get a suitable shape using numerical flow simulation, for instance in Fig. 4.1-2, where the flow field does not contain any separation area, backflow, etc. [6].

Numerical flow modeling of this case could simply predict possible operational troubles at an unsuitable shape of the channel. They can be avoided in the designing period, long time before the construction of complicated and expensive thermal equipment. When it is already built, the problem is practically irremovable.

\section{2. $\operatorname{Rod}$ Burner}

The long rod burner consists from a long tube with many small outlets. It is important to design such arrangement to have the same gas flow (or the same heat output) in each outlet. The essential condition for it is to have the common tube character as a pressure vessel with many small outlets. If the tube diameter is not quite large, then it obtains the character of a conduit, along its length, the pressure is decreasing due to the pressure losses and the pressure gradient for individual outlets is different. Accordingly, the heat output of individual outlets is decreasing, too.

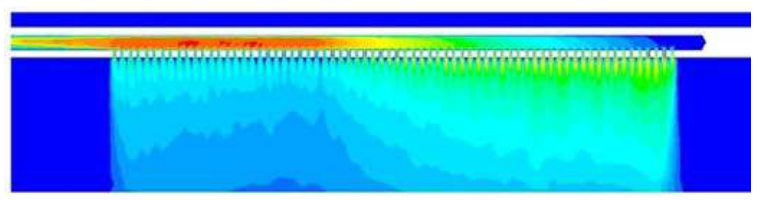

Figure 4.2-1. Pressure field of rod burner and surroundings

Fig. 4.2-1 shows the global overview of the pressure field of the well dimensioned rod burner, Fig. 4.2-2 shows the detail of the velocity field in several adjoining outlets.

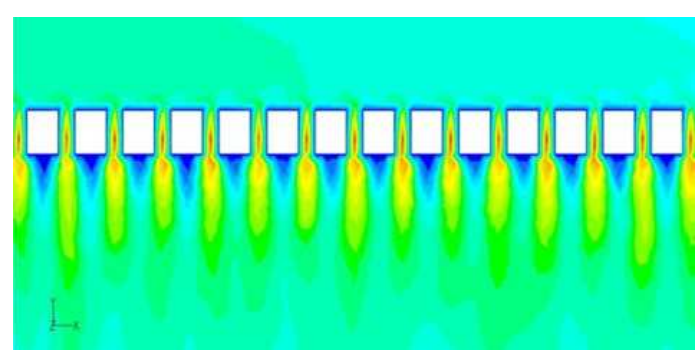

Figure 4.2-2. Velocity field in several outlets in tube wall

\section{References}

[1] K. Adamek, "Problems of industrial air exhausting", WCCM $\mathrm{XI}+\mathrm{ECCM} \mathrm{V} \mathrm{+} \mathrm{ECFD} \mathrm{VI,} \mathrm{Barcelona,} \mathrm{2014.} \mathrm{ISBN}$ 978-84-942844-7-2.

[2] K. Adamek, J. Kolar, P. Půlpan, M. Pustka, "Noise reduction at the fan outlet", Int. conf. on energy, environment, ecosystems and development (EEED 2013), Rhodes Island 2013. ISBN 978-1-61804-202-6.

[3] K. Adamek, J. Kolar, P. Půlpan, M. Pustka, "Noise reduction at the fan outlet", Computational Problems of Engineering, ed. Matsorakis, Springer Verlag, 2014, ISBN 978-1-61804-202-6.

[4] Czech Utility design No. 2013-28182: Device for noise damping of the air flow, 20.01.2014. 
[5] J. Kolar, K. Adamek,"Evaporation and impact flows in CFD", EMT09 (Experimental Fluid mechanics, TU Liberec, Jičin 2009. ISBN 978-80-7372-538-9.
[6] K. Adamek, "Diffuser of large angle of enlargement", Int. conf. Applic. of exp. and numer. meth. in fluid mech. (2000), p. 6-11, University of Zilina. ISBN 80-7100-717-X (in Czech). 